This item was submitted to Loughborough's Research Repository by the author.

Items in Figshare are protected by copyright, with all rights reserved, unless otherwise indicated.

\title{
Bifurcations of phase portraits of pendulum with vibrating suspension point
}

\section{PLEASE CITE THE PUBLISHED VERSION}

http://dx.doi.org/10.1016/j.cnsns.2016.11.003

\section{PUBLISHER}

(c) Elsevier

\section{VERSION}

AM (Accepted Manuscript)

\section{PUBLISHER STATEMENT}

This work is made available according to the conditions of the Creative Commons Attribution-NonCommercialNoDerivatives 4.0 International (CC BY-NC-ND 4.0) licence. Full details of this licence are available at: https://creativecommons.org/licenses/by-nc-nd/4.0/

\section{LICENCE}

CC BY-NC-ND 4.0

\section{REPOSITORY RECORD}

Neishtadt, Anatoly, and K. Sheng. 2019. "Bifurcations of Phase Portraits of Pendulum with Vibrating Suspension Point”. figshare. https://hdl.handle.net/2134/23442. 


\title{
Bifurcations of phase portraits of pendulum with vibrating suspension point
}

\author{
A.I. Neishtadt ${ }^{1,2, *}$, K. Sheng ${ }^{1}$ \\ ${ }^{1}$ Loughborough University, Loughborough, LE11 3TU, UK \\ ${ }^{2}$ Space Research Institute, Moscow, 117997, Russia
}

\begin{abstract}
We consider a simple pendulum whose suspension point undergoes fast vibrations in the plane of motion of the pendulum. The averaged over the fast vibrations system is a Hamiltonian system with one degree of freedom depending on two parameters. We give a complete description of bifurcations of phase portraits of this averaged system.
\end{abstract}

\section{Introduction}

A simple pendulum with vibrating suspension point is a classical problem of perturbation theory. The phenomenon of stabilisation of the upper vertical position of the pendulum by fast vertical vibrations of the suspension point was discovered by A. Stephenson [1, 2]. In these papers the linearisation and reduction to the Mathieu equation is used. The case of inclined vibrations of the suspension point is considered as well. Nonlinear theory was developed by N.N.Bogolyubov [3], who used the averaging method, and by P.L.Kapitsa, who has developed a method of separation of slow and fast motions for this [4, 5] (see also [6]). Different aspects of this problem were discussed in many publications (see, e.g., [7] for a discussion of geometric aspects, [8] for the case of arbitrary frequencies of vibrations, [9] for the case of random vibrations). Generalisations to double- and multiple-link pendulums are contained in $[11,12,13]$. It is noted in [10] that the problem is simplified by using averaging in Hamiltonian form. Such an approach is used, e.g., in [14, 15]. In [14] bifurcations of the phase portraits of the averaged problem for a vertically vibrating suspension point are described.

\footnotetext{
${ }^{*}$ Corresponding author. E-mail addresses: A.Neishtadt@lboro.ac.uk (A.I.Neishtadt), shengkaicheng@yeah.net (K.Sheng)
} 
We consider the case of arbitrary planar vibrations of the suspension point. We use the Hamiltonian approach of [10] to construct the averaged system and give a complete description of bifurcations of its phase portraits. Equations for equilibria of the averaged system in this case are obtained in [16].

\section{Hamiltonian of the problem}

Consider a simple pendulum, Fig. 1, whose suspension point moves in the vertical plane where the pendulum moves. Let $l, m$ be length of the massless rod and mass of the bob for this pendulum. Let $\xi, \eta$ be horizontal and vertical Cartesian coordinates of the suspension point. It is assumed that $\xi, \eta$ are some given functions of time. Denote by $\varphi$ the angle between the pendulum rod and the vertical line straight down.

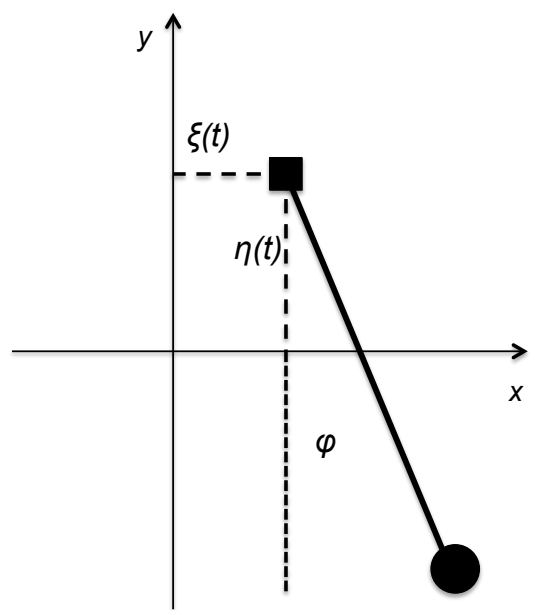

Figure 1. A pendulum with vibrating suspension point.

Then the kinetic and potential energies of the bob are

$$
T=\frac{1}{2}\left[l^{2} \dot{\varphi}^{2}+2 l \dot{\varphi}(\dot{\xi} \cos \varphi+\dot{\eta} \sin \varphi)+\left(\dot{\xi}^{2}+\dot{\eta}^{2}\right)\right], \quad V=m g(\eta-l \cos \varphi) .
$$

Here $g$ is the gravity acceleration. The generalised momentum conjugate to $\varphi$ is

$$
p=\partial T / \partial \dot{\varphi}=m l^{2} \dot{\varphi}+m l(\dot{\xi} \cos \varphi+\dot{\eta} \sin \varphi) \text {. }
$$

Thus

$$
\dot{\varphi}=\frac{p}{m l^{2}}-\frac{(\dot{\xi} \cos \varphi+\dot{\eta} \sin \varphi)}{l}
$$

In order to obtain the Hamiltonian of the problem we should substitute this expression into $T+V$ and subtract terms which do not depend on $p$. Thus we get the Hamiltonian

$$
H=\frac{1}{2}\left(\frac{p^{2}}{m l^{2}}-2 \frac{p(\dot{\xi} \cos \varphi+\dot{\eta} \sin \varphi)}{l}+m(\dot{\xi} \cos \varphi+\dot{\eta} \sin \varphi)^{2}\right)-m g l \cos \varphi
$$




\section{Averaged Hamiltonian}

Assume that $\xi=\varepsilon \tilde{\xi}(\omega t / \varepsilon), \eta=\varepsilon \tilde{\eta}(\omega t / \varepsilon)$, where $\varepsilon$ is a small parameter, $\tilde{\xi}(\cdot), \tilde{\eta}(\cdot)$ are $2 \pi$ periodic functions with zero average. Then $\dot{\xi}, \dot{\eta}$ are values of order 1 . In the system of Hamilton's equations

$$
\dot{\varphi}=\partial H / \partial p, \quad \dot{p}=-\partial H / \partial \varphi .
$$

the right hand side is a fast oscillating function of time. In line with the averaging method [17], for an approximate description of dynamics of variables $\varphi, p$ we average the Hamiltonian $H$ with respect to time $t$. Denote by $A, B, C$ the averages of $\frac{\dot{\xi}^{2}}{2}, \frac{\dot{\eta}^{2}}{2}$, and $\dot{\xi} \dot{\eta}$, respectively. Then the averaged Hamiltonian up to an additive constant is

$$
\bar{H}=\frac{1}{2} \frac{p^{2}}{m l^{2}}+\bar{V}(\varphi), \quad \bar{V}=\bar{V}(\varphi)=\frac{m}{2}[(A-B) \cos 2 \varphi+C \sin 2 \varphi]-m g l \cos \varphi .
$$

The type of the phase portrait of $H$ is determined by the extrema of $\bar{V}$. Effectively, it depends on two parameters, $(B-A) /(g l)$ and $C /(g l)$. Our goal is to draw the bifurcation diagram of the problem. We have to determine a partition of the plane of parameters by curves into domains such that the type of the phase portrait changes only across these curves.

The function $\bar{V}$ is invariant under the transformation $C \rightarrow-C, \varphi \rightarrow-\varphi$. Thus, without loss of generality, in calculations below we assume that $C \geq 0$.

\section{Particular cases}

\subsection{Case $C=0$}

This is the case considered by Stephenson, Bogolyubov, and Kapitsa under an additional assumption that $A=0$. Phase portraits for this case are presented in [14] (for $A=0$ ).

If $\left|\frac{2(B-A)}{g l}\right|<1$, then $\bar{V}$ has a minimum at $\varphi=0$ and a maximum at $\varphi=\pi$. The phase portrait is shown in Fig. 2.

If $\frac{2(B-A)}{g l}>1$, then $\bar{V}$ has minima at $\varphi=0$ and $\varphi=\pi$, and maxima at $\varphi= \pm \arccos \frac{g l}{2(A-B)}$.

The phase portrait is shown in Fig. 3. In this case both the upper and lower vertical equilibria of the pendulum are stable.

If $\frac{2(B-A)}{g l}<-1$, then $\bar{V}$ has maxima at $\varphi=0$ and $\varphi=\pi$, and minima at $\varphi= \pm \arccos \frac{g l}{2(A-B)}$. The phase portrait is shown in Fig. 4. In this case both the lower and upper vertical equilibria of the pendulum are unstable. 


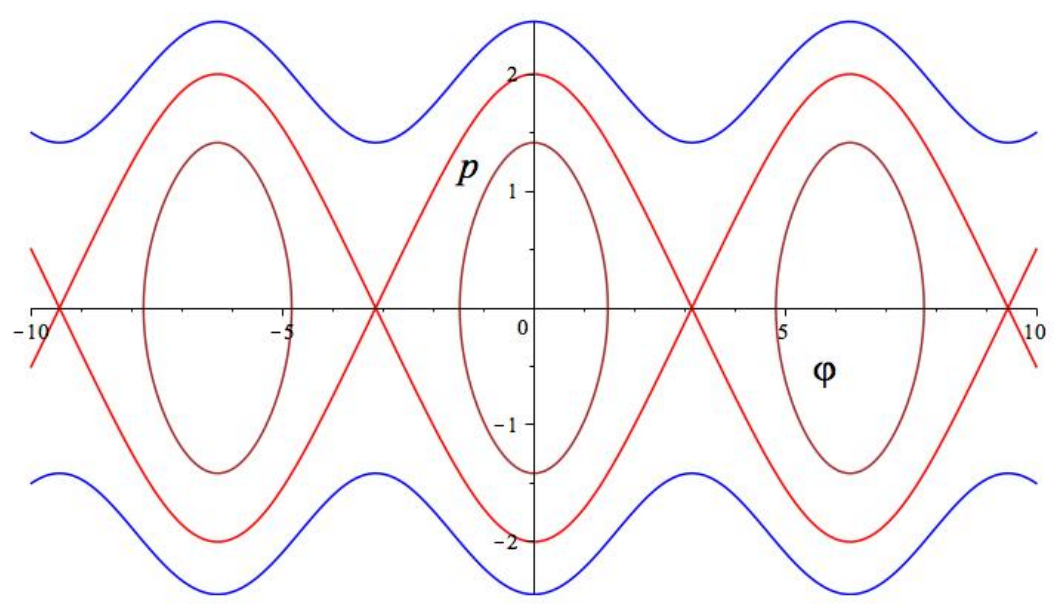

Figure 2. $\left|\frac{2(B-A)}{g l}\right|<1$.

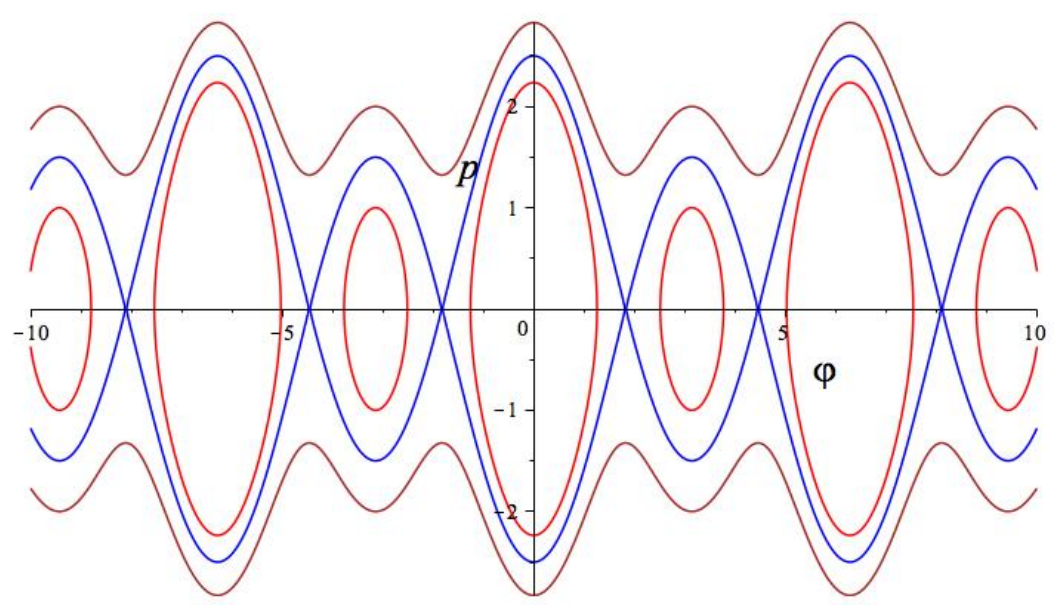

Figure 3. $\frac{2(B-A)}{g l}>1$.

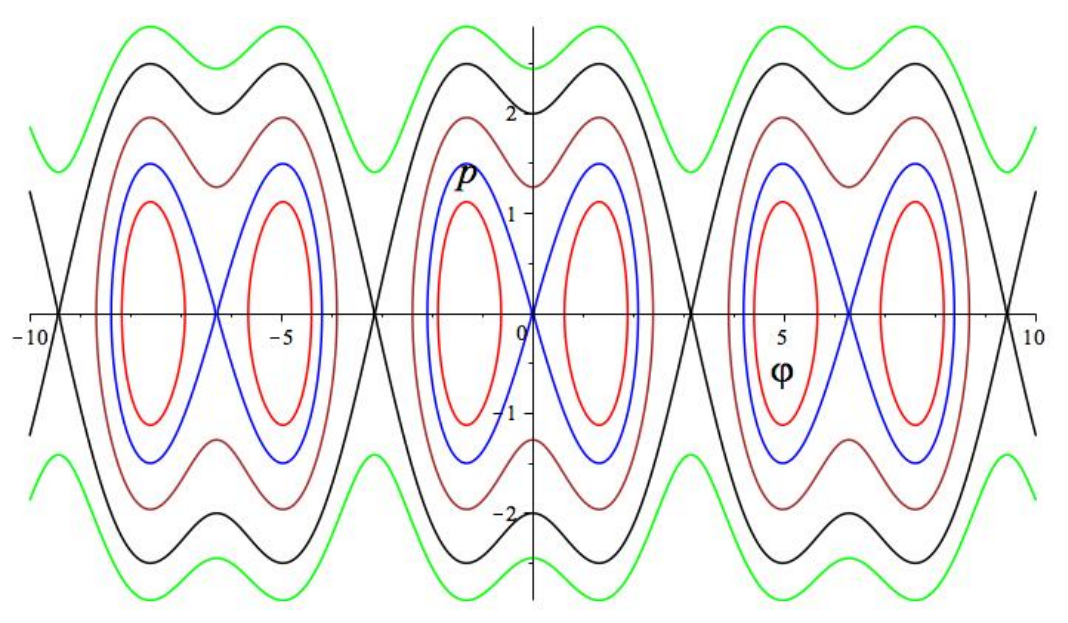

Figure 4. $\frac{2(B-A)}{g l}<-1$. 
We observe a coalescence of two maxima and a minimum of the potential $\bar{V}$ at $\frac{2(B-A)}{g l}=1$ and that of two minima and a maximum of $\bar{V}$ at $\frac{2(B-A)}{g l}=-1$.

\subsection{Case $A=B$}

The equation for extrema of $\bar{V}$ is

$$
\partial \bar{V} / \partial \varphi=m(C \cos 2 \varphi+g l \sin \varphi)=0
$$

which implies

$$
\sin \varphi=\frac{g l \pm \sqrt{g^{2} l^{2}+8 C^{2}}}{4 C} .
$$

For the sign "-" in this formula and $C>0$ the value in the right hand side is in the interval $(-1,0)$. Thus we have two solutions

$$
\varphi_{-, 1}=\arcsin \left(\frac{g l-\sqrt{g^{2} l^{2}+8 C^{2}}}{4 C}\right), \varphi_{-, 2}=\pi-\varphi_{-, 1} .
$$

They correspond to a minimum and a maximum of $\bar{V}$, respectively.

If $C>l g$ then for the sign "+" in (1) the value in the right hand side is in the interval $(0,1)$. In this case we have two additional solutions

$$
\varphi_{+, 1}=\arcsin \left(\frac{g l+\sqrt{g^{2} l^{2}+8 C^{2}}}{4 C}\right), \varphi_{+, 2}=\pi-\varphi_{+, 1} .
$$

They correspond to a maximum and a minimum of $\bar{V}$, respectively.

The phase portrait for the case $-l g<C<l g$ has the form shown in Fig. 2 (but it is in general not symmetric with respect to the axis $\varphi=0$, cf. Fig. 8). Phase portraits for the cases $C>\lg$ and $C<-l g$ are shown in Fig. 5 and Fig. 6, respectively.

\section{General case}

We will build a partition of the parameter plane of the problem into domains corresponding to different types of phase portraits of the averaged system. The boundaries of these domains are critical curves. There are two types of critical curves: 


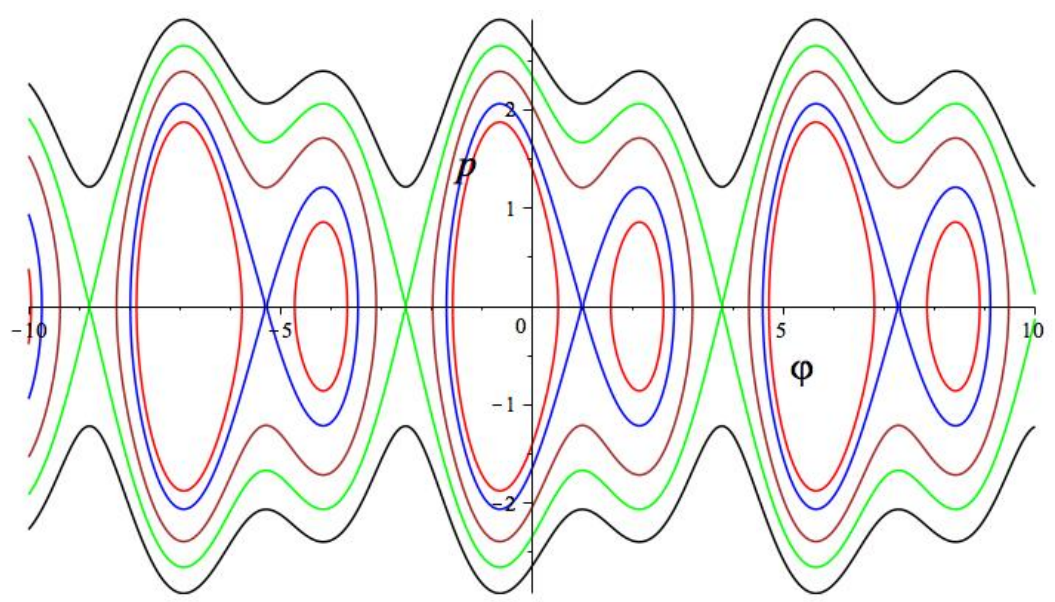

Figure 5. $C>l g$.

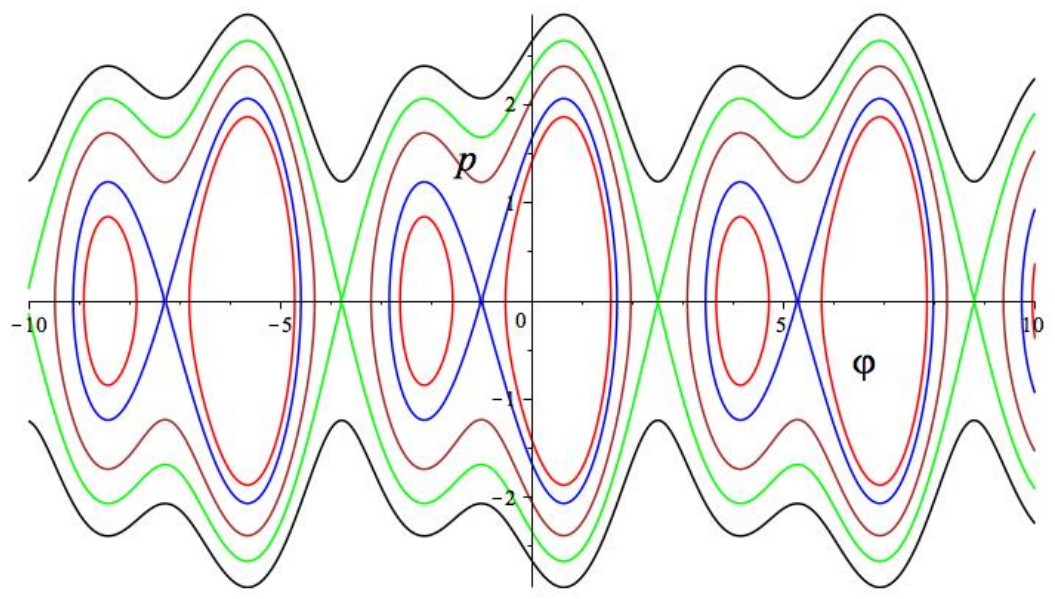

Figure 6. $C<-l g$. 
- Curves corresponding to degenerate equilibria: first and second derivatives of $\bar{V}$ vanish for parameters on these curves. The number of equilibria changes at crossing such a curve in the plane of parameters.

- Curves such that the function $\bar{V}$ has the same value at two different saddle equilibria for parameters on these curves. The number of equilibria remains the same at crossing such a curve in the plane of parameters. Curves of the second type locally separate adjacent regions with different behavior of separatrices.

\subsection{Critical curves corresponding to degenerate equilibria}

These curves correspond to values of parameters such that

$$
\begin{array}{r}
\partial \bar{V} / \partial \varphi=m[(B-A) \sin 2 \varphi+C \cos 2 \varphi+g l \sin \varphi]=0, \\
\partial^{2} \bar{V} / \partial \varphi^{2}=m[2(B-A) \cos 2 \varphi-2 C \sin 2 \varphi+g l \cos \varphi]=0
\end{array}
$$

which is equivalent to

$$
\begin{gathered}
\frac{B-A}{g l} \sin 2 \varphi+\frac{C}{g l} \cos 2 \varphi+\sin \varphi=0, \\
\frac{2(B-A)}{g l} \cos 2 \varphi-\frac{2 C}{g l} \sin 2 \varphi+\cos \varphi=0 .
\end{gathered}
$$

This system is invariant with respect to the change $B-A \rightarrow-(B-A), \varphi \rightarrow \pi-\varphi$. Thus, without loss of generality, in this subsection we assume that $B-A \geq 0$. As agreed earlier, we also assume that $C>0$.

The system (2) can be considered as a system of two linear equations for unknown $(B-A) /(g l)$ and $C /(g l)$. Solving it for these unknown we get

$$
\begin{aligned}
\frac{B-A}{g l} & =-\sin \varphi \sin 2 \varphi-\frac{1}{2} \cos \varphi \cos 2 \varphi, \\
\frac{C}{g l} & =-\sin \varphi \cos 2 \varphi+\frac{1}{2} \cos \varphi \sin 2 \varphi .
\end{aligned}
$$

An a bit more compact form of these relations is

$$
\begin{aligned}
\frac{B-A}{g l} & =\cos ^{3} \varphi-\frac{3}{2} \cos \varphi, \\
\frac{C}{g l} & =\sin ^{3} \varphi .
\end{aligned}
$$

These relations give a parametric representation with the parameter $\varphi$ of the critical curve $\Gamma$ corresponding to degenerate equilibria. This curve is shown in Fig. 7. 


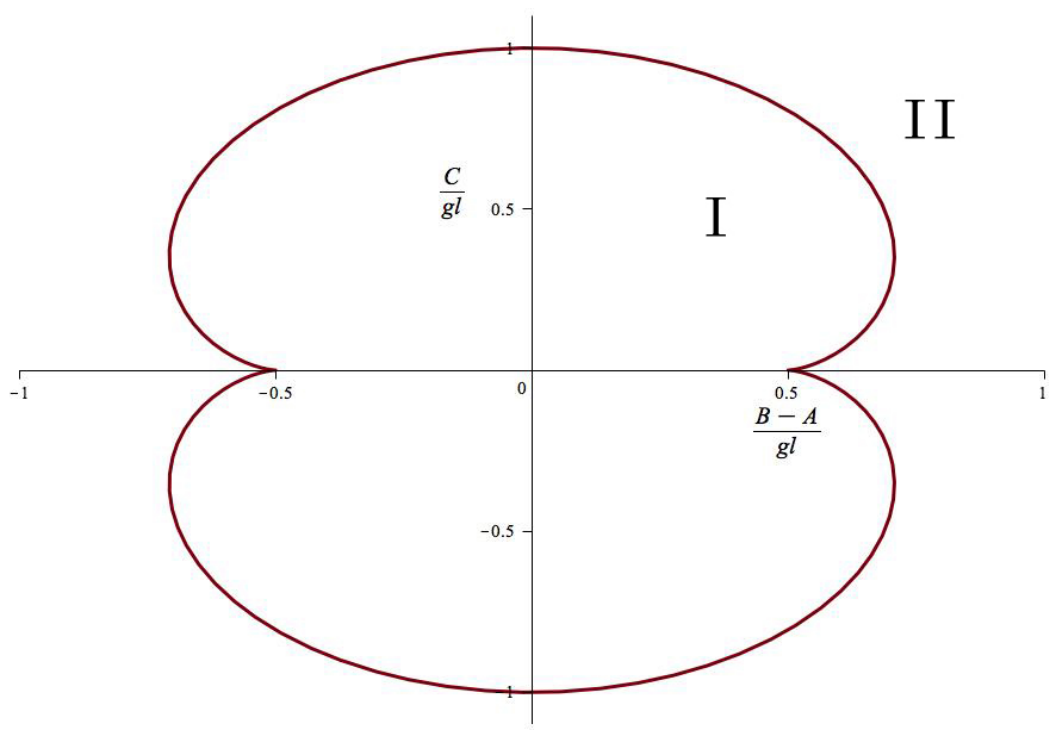

Figure 7. Bifurcation curve corresponding to degenerate equilibria.

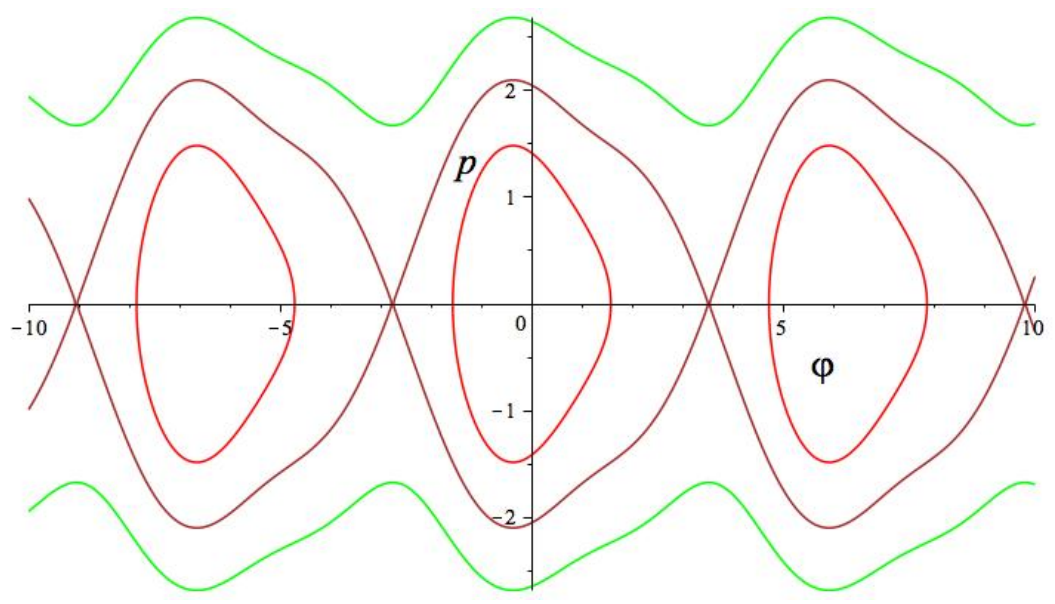

Figure 8. Phase portrait for domain $I$. 
The pendulum has 2 equilibria if parameters are in domain $I$ (Fig. 8), and 4 equilibria if parameters are in domain $I I$ (Figs. 9, 10).

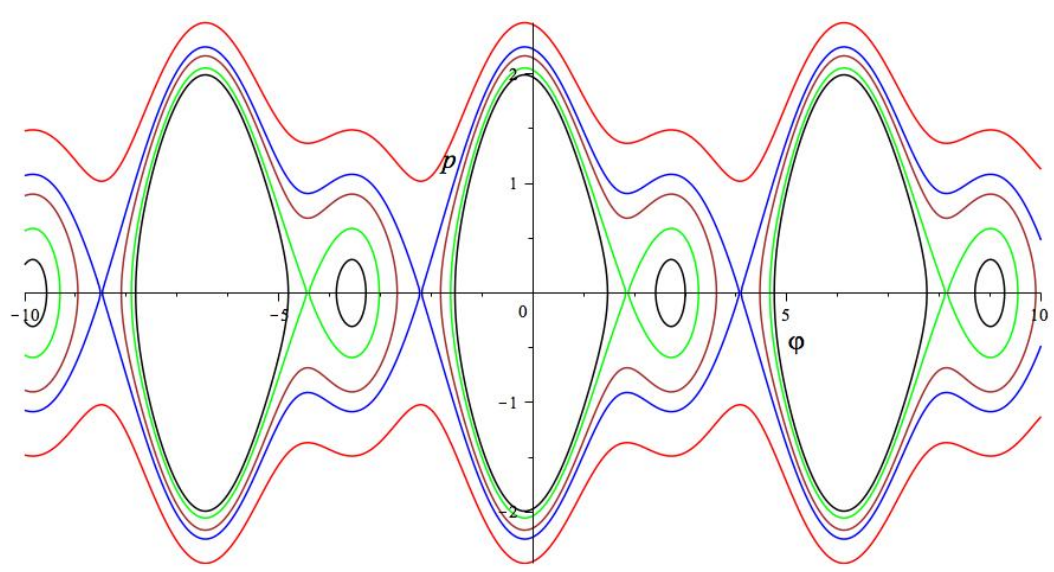

Figure 9. Phase portrait for domain $I I, C>0$.

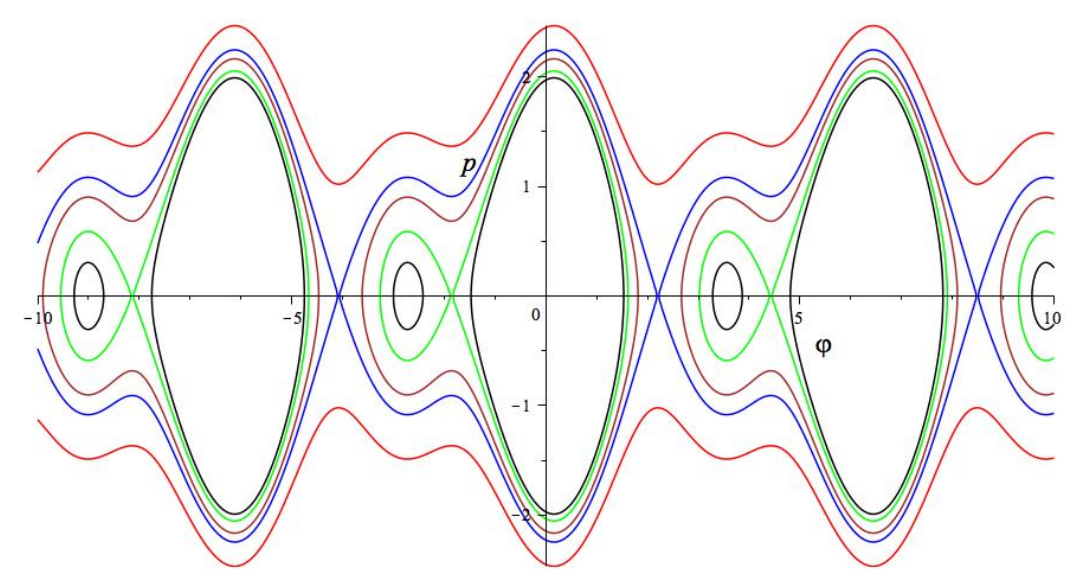

Figure 10. Phase portrait for domain $I I, C<0$.

The curve $\Gamma$ has two cusp points. Regular points of $\Gamma$ correspond to centre-saddle bifurcations: centre and saddle equilibria coalesce and disappear. Let us prove that these bifurcations are not degenerate, i.e., at regular points of $\Gamma$, (a) $\partial^{3} \bar{V} / \partial \varphi^{3} \neq 0$ and (b) the derivative of $\partial \bar{V} / \partial \varphi$ along a direction transversal to $\Gamma$ is different from 0 (after calculation of derivatives one should substitute the equilibrium value of $\varphi$ from (2) into obtained expressions). 
For (a), we have

$$
\frac{1}{m g l} \frac{\partial^{3} \bar{V}}{\partial \varphi^{3}}=-\frac{4(B-A)}{g l} \sin 2 \varphi-\frac{4 C}{g l} \cos 2 \varphi-\sin \varphi=3 \sin \varphi \neq 0
$$

at regular points of $\Gamma$ (we used the first equality in (2) here).

For (b), we note that the parametric representation (3) of $\Gamma$ implies that the vector

$$
\mathbf{u}=\left(-3 \cos ^{2} \varphi \sin \varphi+\frac{3}{2} \sin \varphi, 3 \sin ^{2} \varphi \cos \varphi\right)
$$

is tangent to $\Gamma$. Then

$$
\mathbf{u}^{\perp}=\left(3 \sin ^{2} \varphi \cos \varphi, 3 \cos ^{2} \varphi \sin \varphi-\frac{3}{2} \sin \varphi\right)
$$

is a vector transversal to $\Gamma$. For the directional derivative of $\partial \bar{V} / \partial \varphi$ along $\mathbf{u}^{\perp}$ we get

$$
\begin{aligned}
\frac{1}{m g l} \nabla_{\mathbf{u}^{\perp}}\left(\frac{\partial \bar{V}}{\partial \varphi}\right) & =3 \sin ^{2} \varphi \cos \varphi \sin 2 \varphi+\left(3 \cos ^{2} \varphi \sin \varphi-\frac{3}{2} \sin \varphi\right) \cos 2 \varphi \\
& =\frac{3}{2} \sin \varphi\left(2 \sin \varphi \cos \varphi \sin 2 \varphi+\left(2 \cos ^{2} \varphi-1\right) \cos 2 \varphi\right)=\frac{3}{2} \sin \varphi \neq 0
\end{aligned}
$$

at regular points of $\Gamma$. Thus, bifurcations at regular points of $\Gamma$ are not degenerate.

At the cusp points of $\Gamma$ we have $\partial \bar{V} / \partial \varphi=\partial^{2} \bar{V} / \partial \varphi^{2}=\partial^{3} \bar{V} / \partial \varphi^{3}=0, \partial^{4} \bar{V} / \partial \varphi^{4}= \pm 3 m g l \neq 0$ at the equilibria ( \pm for the left and right cusp points, respectively). Near cusp points the curve $\Gamma$ has the standard form a of semi-cubic parabola:

$$
\left\{\begin{array} { l } 
{ \frac { B - A } { g l } = - \frac { 1 } { 2 } - \frac { 3 } { 4 } \varphi ^ { 2 } + \ldots } \\
{ \frac { C } { g l } = \varphi ^ { 3 } + \ldots }
\end{array} \quad \text { or } \quad \left\{\begin{array}{l}
\frac{B-A}{g l}=\frac{1}{2}+\frac{3}{4}(\varphi-\pi)^{2}+\ldots \\
\frac{C}{g l}=-(\varphi-\pi)^{3}+\ldots
\end{array}\right.\right.
$$

for the left and the right cusp points, respectively. This implies that the normal forms of the Hamiltonian near the cusp points are of a generic form presented, e.g., in [18], example 2.6, and thus the bifurcations at cusp points are not degenerate.

\subsection{Critical curves corresponding to saddle equilibria with equal values of potential energy}

Let $\varphi_{1}$ and $\varphi_{2}$ be coordinates of such equilibria, $\varphi_{1} \neq \varphi_{2} \bmod 2 \pi$. We should have

$$
\partial \bar{V}\left(\varphi_{1}\right) / \partial \varphi=0, \partial \bar{V}\left(\varphi_{2}\right) / \partial \varphi=0, \bar{V}\left(\varphi_{1}\right)=\bar{V}\left(\varphi_{2}\right)
$$


or, in the explicit form,

$$
\begin{aligned}
\frac{B-A}{g l} \sin 2 \varphi_{1} & +\frac{C}{g l} \cos 2 \varphi_{1}+\sin \varphi_{1}=0 \\
\frac{B-A}{g l} \sin 2 \varphi_{2} & +\frac{C}{g l} \cos 2 \varphi_{2}+\sin \varphi_{2}=0 \\
-\frac{B-A}{g l}\left(\cos 2 \varphi_{1}-\cos 2 \varphi_{2}\right) & +\frac{C}{g l}\left(\sin 2 \varphi_{1}-\sin 2 \varphi_{2}\right)-2\left(\cos \varphi_{1}-\cos \varphi_{2}\right)=0 .
\end{aligned}
$$

As $\varphi_{1} \neq \varphi_{2} \bmod 2 \pi$, we can divide the last equation by $4 \sin \frac{\varphi_{1}-\varphi_{2}}{2}$. It reduces to

$$
\frac{B-A}{g l} \sin \left(\varphi_{1}+\varphi_{2}\right) \cos \frac{\varphi_{1}-\varphi_{2}}{2}+\frac{C}{g l} \cos \frac{\varphi_{1}-\varphi_{2}}{2} \cos \left(\varphi_{1}+\varphi_{2}\right)+\sin \frac{\varphi_{1}+\varphi_{2}}{2}=0 .
$$

The first two equations in (4) can be considered as a system of two linear equations for unknown $(B-A) /(g l)$ and $C /(g l)$. The determinant of this system is

$$
D=\sin 2\left(\varphi_{1}-\varphi_{2}\right) .
$$

We should consider two cases: $D=0$ and $D \neq 0$.

In the case $D \neq 0$ we solve first two equations in (4) for $(B-A) /(g l)$ and $C /(g l)$ and get

$$
\begin{aligned}
\frac{B-A}{g l} & =\frac{-\sin \varphi_{1} \cos 2 \varphi_{2}+\sin \varphi_{2} \cos 2 \varphi_{1}}{\sin 2\left(\varphi_{1}-\varphi_{2}\right)} \\
\frac{C}{g l} & =\frac{\sin \varphi_{1} \sin 2 \varphi_{2}-\sin \varphi_{2} \sin 2 \varphi_{1}}{\sin 2\left(\varphi_{1}-\varphi_{2}\right)}
\end{aligned}
$$

These relations can be rewritten in the form

$$
\begin{aligned}
\frac{B-A}{g l} & =\frac{2 \sin \frac{\varphi_{1}-\varphi_{2}}{2} \cos \frac{\varphi_{1}+\varphi_{2}}{2}\left(\cos \left(\varphi_{1}+\varphi_{2}\right)-\cos \left(\varphi_{1}-\varphi_{2}\right)-1\right)}{\sin 2\left(\varphi_{1}-\varphi_{2}\right)} \\
\frac{C}{g l} & =\frac{2 \sin \frac{\varphi_{1}-\varphi_{2}}{2} \sin \frac{\varphi_{1}+\varphi_{2}}{2}\left(-\cos \left(\varphi_{1}+\varphi_{2}\right)+\cos \left(\varphi_{1}-\varphi_{2}\right)\right)}{\sin 2\left(\varphi_{1}-\varphi_{2}\right)}
\end{aligned}
$$

or, after a cancellation, in the form

$$
\begin{aligned}
\frac{B-A}{g l} & =\frac{\cos \frac{\varphi_{1}+\varphi_{2}}{2}\left(\cos \left(\varphi_{1}+\varphi_{2}\right)-\cos \left(\varphi_{1}-\varphi_{2}\right)-1\right)}{2 \cos \left(\varphi_{1}-\varphi_{2}\right) \cos \frac{\varphi_{1}-\varphi_{2}}{2}}, \\
\frac{C}{g l} & =\frac{\sin \frac{\varphi_{1}+\varphi_{2}}{2}\left(-\cos \left(\varphi_{1}+\varphi_{2}\right)+\cos \left(\varphi_{1}-\varphi_{2}\right)\right)}{2 \cos \left(\varphi_{1}-\varphi_{2}\right) \cos \frac{\varphi_{1}-\varphi_{2}}{2}} .
\end{aligned}
$$

Now we substitute these relations into equation (5), and, assuming that $\varphi_{1} \neq-\varphi_{2} \bmod 2 \pi$, divide the obtained equation by $\sin \frac{\varphi_{1}+\varphi_{2}}{2}$. We get

$$
\begin{aligned}
& \frac{\cos ^{2} \frac{\varphi_{1}+\varphi_{2}}{2}\left(\cos \left(\varphi_{1}+\varphi_{2}\right)-\cos \left(\varphi_{1}-\varphi_{2}\right)-1\right)}{\cos \left(\varphi_{1}-\varphi_{2}\right)} \\
& +\frac{\left(-\cos \left(\varphi_{1}+\varphi_{2}\right)+\cos \left(\varphi_{1}-\varphi_{2}\right)\right)}{2 \cos \left(\varphi_{1}-\varphi_{2}\right)} \cos \left(\varphi_{1}+\varphi_{2}\right)+1=0
\end{aligned}
$$


which is equivalent to

$$
\begin{aligned}
& \left(1+\cos \left(\varphi_{1}+\varphi_{2}\right)\right)\left(\cos \left(\varphi_{1}+\varphi_{2}\right)-\cos \left(\varphi_{1}-\varphi_{2}\right)-1\right) \\
& +\quad\left(-\cos \left(\varphi_{1}+\varphi_{2}\right)+\cos \left(\varphi_{1}-\varphi_{2}\right)\right) \cos \left(\varphi_{1}+\varphi_{2}\right)+2 \cos \left(\varphi_{1}-\varphi_{2}\right)=0
\end{aligned}
$$

and then equivalent to

$$
\cos \left(\varphi_{1}-\varphi_{2}\right)=1
$$

which can not be satisfied for $\varphi_{1} \neq \varphi_{2} \bmod 2 \pi$.

Thus, the only remaining possibilities are related to the special cases $D=\sin 2\left(\varphi_{1}-\varphi_{2}\right)=0$ and $\varphi_{1}=-\varphi_{2} \bmod 2 \pi$. It is easy to check that each of these relations together with relations (4) implies $C=0$. As $\varphi_{1}$ and $\varphi_{2}$ should correspond to different saddles, we should consider the domain were there are four equilibria. Thus, possible bifurcational curves are rays

$\left\{C=0, \frac{2(B-A)}{g l}>1\right\}$ and $\left\{C=0, \frac{2(B-A)}{g l}<-1\right\}$. The equilibria with equal values of potential energy are saddles for the first and centres for the second of these rays (see Figs. 3 and 4 ). Thus the only bifurcational curve of the considered type is the ray $\left\{C=0, \frac{2(B-A)}{g l}>1\right\}$.

\subsection{Bifurcation diagram}

The partition of the plane of parameters into domains with qualitatively different phase portraits is shown in Fig. 11. The pendulum has 2 equilibria if parameters are in domain $I$ (phase portrait in Fig. 8), and 4 equilibria if parameters are in domain $I I$ (phase portrait in Fig. 4 for $C=0$, Fig. 9 for $C>0$ and Fig. 10 for $C<0$; these phase portraits are topologically the same. The boundary $\Gamma$ between these domains is the curve of degenerate equilibria. The ray $\left\{C=0, \frac{2(B-A)}{g l}>1\right\}$ corresponds to phase portraits with a heteroclinic trajectory (Fig. 3). Passage through this ray transforms the phase portrait of Fig. 9 via that of Fig. 3 to that of Fig. 10. Upon approaching the curve of degenerate equilibria from the domain $I I$ at $C>0$ (respectively $C<0$ ), the right (respectively, the left) loop of eight-shaped separatrix shrinks to a point and disappears.

\section{Conclusion}

Our study in this paper provides a complete description of bifurcations of phase portraits for a pendulum with vibrating suspension point in the approximation of the averaging method. The relation of such a description to dynamics in the exact (not averaged) problem for high-frequency oscillations is discussed, e.g., in [15], Sect. 6.3.3.B. One should consider the Poincaré return map for the plane $\varphi, p\left(\right.$ Poincaré section $\left.t=0 \bmod \frac{2 \pi \varepsilon}{\omega}\right)$. Fixed points of this 


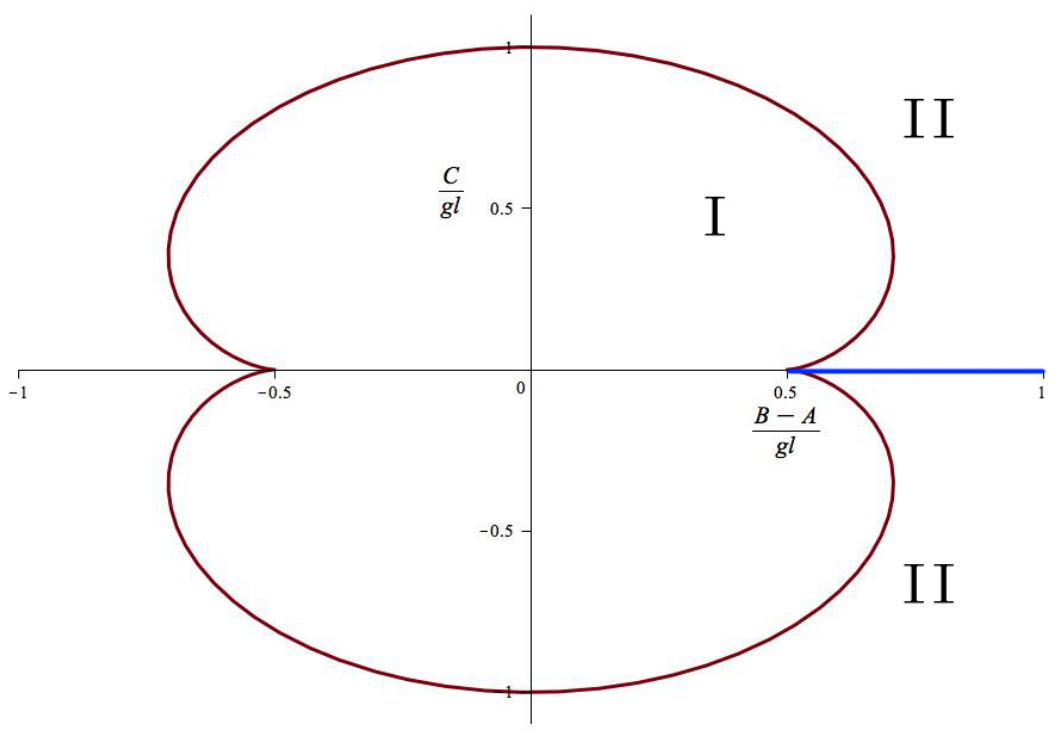

Figure 11. Partition of the parameter plane.

map are close to equilibria of the averaged system. The plane $\varphi, p$ is filled by invariant curves of this map up to a remainder of a measure which is exponentially small in the perturbation parameter $\varepsilon$. Stable and unstable manifolds of hyperbolic fixed points of the return map are split, but this splitting is exponentially small. These manifolds are close to separatrices of the averaged system. Thus, the study of the averaged system provides considerable information about dynamics of the exact (not averaged) problem.

\section{Acknowledgement}

The authors are very grateful to the anonymous referee for a constructive criticism and useful suggestions. 


\section{References}

[1] Stephenson A. On induced stability. Philosophical Magazine Series 6 1908; 15: 233-6.

[2] Stephenson A. On a new type of dynamical stability. Mem Proc Manch Lit Phil Soc 1908; 52 (8): 1-10.

[3] Bogolyubov NN. Perturbation theory in nonlinear mechanics. In: Collection of papers of Inst Constuct Mekh Akad Nauk UkrSSR 1950; 14: 9-34 (in Russian).

[4] Kapitsa PL. Dynamic stability of a pendulum with oscillating point of suspension. Sov. Phys. JETP 1951; 21: 588-597 (in Russian), see also Collected papers of P. L. Kapitza, vol. 2, 714-725. London: Pergamon; 1965.

[5] Kapitsa PL. A pendulum with vibrating point of suspension. Usp Phys Nauk 1951; 44: 7-20 (in Russian).

[6] Landau LD, Lifshitz EM. Course of theoretical physics, Vol. 1: Mechanics. Oxford: Pergamon; 1988.

[7] Levi M. Geometry of Kapitsa's potential. Nonlinearity 1998; 11: 1365-8.

[8] Bardin BS, Markeyev AP. On the stability of equilibrium of a pendulum with vertical oscillations of its suspension point. J Appl Math Mech 1995; 59: 879-86.

[9] Ovseyevich AI. The stability of an inverted pendulum when there are rapid random oscillations of the suspension point. J Appl Math Mech 2006; 70: 761-8.

[10] Burd VSh, Matveev VN. Asymptotic methods on an infinite interval in problems of nonlinear mechanics. Yaroslavl': Yaroslavl' Univ; 1985 (in Russian).

[11] Stephenson A. On induced stability. Philosophical Magazine Series 6 1909; 17: 765-6.

[12] Acheson D. A pendulum theorem. Proc R Soc London 1993; 433: 239-45.

[13] Kholostova O. On the motions of a double pendulum with vibrating suspension point. Mechanics of Solids 2009; 44: 184-97.

[14] Treschev D. Some aspects of finite-dimensional Hamiltonian dynamics. In: Craig W (editor). Hamiltonian dynamical systems and applications, 1-19. Dordrecht: Springer; 2008.

[15] Arnold VI, Kozlov VV, Neishtadt AI. Mathematical aspects of classical and celestial mechanics. Berlin: Springer; 2006.

[16] Akulenko LD. Asymptotic analysis of dynamical systems subjected to high-frequency interactions. J Appl Math Mech. 1994; 58(3): 393-402.

[17] Bogoliubov NN, Mitropolsky YA. Asymptotic methods in the theory of non-linear oscillations. New York: Gordon and Breach Sci Publ; 1961.

[18] Hanßmann H. Local and semi-local bifurcations in Hamiltonian systems - Results and examples. Lecture Notes in Mathematics 1893. Berlin: Springer; 2007. 